\title{
Metastable solitonic states in the strained itinerant helimagnet FeGe
}

\author{
Victor Ukleev $\odot,{ }^{1,2,{ }^{*}}$ Yuichi Yamasaki $\odot,{ }^{1,3,4}$ Oleg Utesov $\odot,{ }^{5,6,7}$ Kiyou Shibata $\odot,{ }^{1, \dagger}$ Naoya Kanazawa, ${ }^{8}$ Nicolas Jaouen $\odot,{ }^{9}$ \\ Hironori Nakao ${ }^{10},{ }^{10}$ Yoshinori Tokura, ${ }^{1,11,12}$ and Taka-hisa Arima ${ }^{1,13}$ \\ ${ }^{1}$ RIKEN Center for Emergent Matter Science (CEMS), Wako 351-0198, Japan \\ ${ }^{2}$ Laboratory for Neutron Scattering and Imaging (LNS), Paul Scherrer Institute (PSI), CH-5232 Villigen, Switzerland \\ ${ }^{3}$ Research and Services Division of Materials Data and Integrated System (MaDIS), National Institute for Materials Science (NIMS), \\ Tsukuba 305-0047, Japan \\ ${ }^{4}$ PRESTO, Japan Science and Technology Agency (JST), Kawaguchi 332-0012, Japan \\ ${ }^{5}$ Petersburg Nuclear Physics Institute NRC “Kurchatov Institute," Gatchina, Saint-Petersburg 188300, Russia \\ ${ }^{6}$ St. Petersburg State University, 7/9 Universitetskaya nab., St. Petersburg 199034, Russia \\ ${ }^{7}$ St. Petersburg Academic University-Nanotechnology Research and Education Centre of the Russian Academy of Sciences, \\ 194021 St. Petersburg, Russia \\ ${ }^{8}$ Department of Applied Physics and Quantum-Phase Electronics Center (QPEC), University of Tokyo, Tokyo 113-8656, Japan \\ ${ }^{9}$ Synchrotron SOLEIL, Saint-Aubin, Boîte Postale 48, 91192 Gif-sur-Yvette Cedex, France \\ ${ }^{10}$ Condensed Matter Research Center and Photon Factory, Institute of Materials Structure Science, High Energy Accelerator Research \\ Organization, Tsukuba 305-0801, Japan \\ ${ }^{11}$ Department of Applied Physics, University of Tokyo, Tokyo 113-8656, Japan \\ ${ }^{12}$ Tokyo College, University of Tokyo, Tokyo 113-8656, Japan \\ ${ }^{13}$ Department of Advanced Materials Science, University of Tokyo, Kashiwa 277-8561, Japan
}

(Received 26 April 2020; revised 22 June 2020; accepted 24 June 2020; published 10 July 2020)

\begin{abstract}
Tensile strain is a promising tool for the creation and manipulation of magnetic solitonic textures in chiral helimagnets via tunable control of magnetic anisotropy and Dzyaloshinskii-Moriya interaction. Here, by using in situ resonant small-angle x-ray scattering, we demonstrate that skyrmion and chiral soliton lattices can be achieved as metastable states in FeGe lamella as distinct states under tensile strain and magnetic fields in various orientations with respect to the deformation. The small-angle scattering data can be well accounted for in the framework of the analytical model for a soliton lattice. By using the experimental results and analytical theory, the unwinding of metastable skyrmions in a perpendicular magnetic field as shown by a small-angle scattering experiment was analyzed via micromagnetic simulation.
\end{abstract}

DOI: 10.1103/PhysRevB.102.014416

\section{INTRODUCTION}

Antisymmetric Dzyaloshinskii-Moriya (DM) [1,2] and Heisenberg exchange interactions in cubic B20 chiral magnets $(\mathrm{MnSi}, \mathrm{FeGe}, \mathrm{FeCoSi})$ result in a helical ground state [3] in which the magnetic moments in the neighboring atomic layers maintain a fixed propagating angle, thus forming a spin helix with fixed chirality [Fig. 1(a)]. The helical wavelength $\lambda=4 \pi A_{\mathrm{ex}} / D$ is determined by the ratio of the exchange stiffness $A_{\mathrm{ex}}$ and the Dzyaloshinskii constant $D$. At zero field, the propagation vector of the helix $\mathbf{q}$ is imposed by cubic anisotropy and typically encompassed along $\langle 111\rangle-(\mathrm{MnSi}$, $\mathrm{FeGe})$ or $\langle 100\rangle$-equivalent $\left(\mathrm{MnGe}, \mathrm{Cu}_{2} \mathrm{OSeO}_{3}\right)$ axes [3]. Application of a magnetic field parallel to the $\mathbf{q}$-vector results in canting of the magnetic moments toward the field direction, and the helical texture transforms to a conical state [Fig. 1(b)]. Further increments of the magnetic field lead to the transition from the conical to the induced ferromagnetic state. In cubic

\footnotetext{
*victor.ukleev@psi.ch

${ }^{\dagger}$ Present address: Institute of Industrial Science, The University of Tokyo, Tokyo 1538505, Japan.
}

B20 helimagnets, the orientation of the helical $q$-vector is determined by the relatively weak cubic anisotropy, and it can be rotated by a small magnetic field. In the case of strong uniaxial anisotropy, which can be caused, for example, by the compressive or tensile strain, a moderate magnetic field applied perpendicular to the helical propagation axis can deform the proper screw magnetic modulation into a chiral soliton lattice (CSL) [4,5] [Fig. 1(c)]. Typically, a CSL appears in the uniaxial chiral magnets due to the interplay between easy-axis type anisotropy, DM interaction, and an external magnetic field. Recently, interest in magnetic CSLs has been increased due to the theoretical predictions of driving magnetic kinks by electric current and crossed magnetic fields $[6,7]$, and the nontrivial dynamics of the solitons in the $\mathrm{GHz}$ frequency range [8,9]. Following the theoretical conceptions, several experimental works have unambiguously demonstrated the formation and manipulation of a CSL in a prototypical uniaxial chiral helimagnet $\mathrm{CrNb}_{3} \mathrm{~S}_{6}$ [10-13].

Another peculiar magnetic structure that appears in the chiral helimagnets is a skyrmion crystal (SkX), a hexagonally ordered array of topologically protected chiral magnetic vortices [14,15] [Fig. 1(d)]. Recently, Shibata et al. have shown that the SkX and individual skyrmions can be deformed by the 


\section{(a) Helical state}

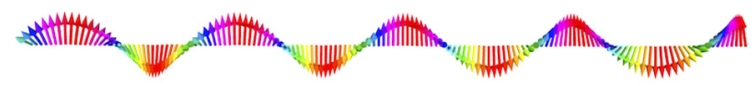

(b) Conical state

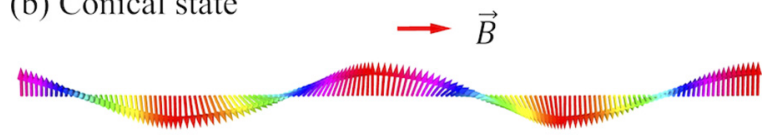

(c) Chiral soliton lattice

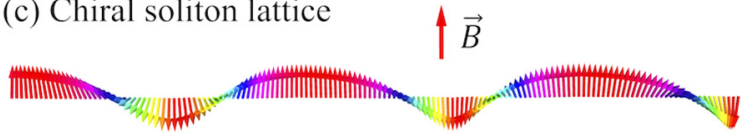

(d) Skyrmion lattice

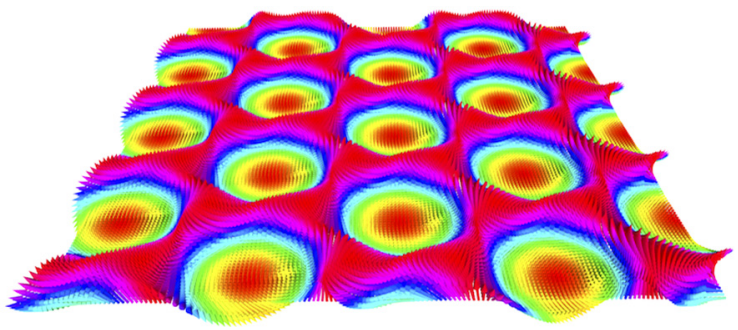

FIG. 1. Schematic illustration of spin configurations in a helical state (a), a conical state (b), a chiral soliton lattice (c), and a skyrmion lattice (d).

tensile strain induced by the lattice mismatch between FeGe lamella and silicon substrate, and this phenomenon was explained by the anisotropic change of the DM interaction [16]. Furthermore, transformation of the magnetic ground state of $\mathrm{Cu}_{2} \mathrm{OSeO}_{3}$ from proper-screw structure to CSL upon application of the tensile strain, also accompanied by the deformation of the SkX, has been demonstrated by Okamura et al. [17]. In the latter case, the transformation of the ground state was explained by the change in magnetic anisotropy induced by the strain. Recently, a large enhancement of the SkX stability in the chemically strained bulk B20 helimagnet MnSi has been demonstrated [18]. Tensile strain induced by the lattice mismatch between the B20 crystal epilayers and the substrate has also been imposed in the thin-film samples of $\mathrm{MnSi}$ and FeGe [19-21]. The strain effect may either extend [19,20] or destabilize the skyrmion phase, favoring the formation of the CSL [22-24]. Therefore, these induced uniaxial anisotropy effects on chiral helimagnets provide a fertile ground for investigation of the interplay between magnetic interactions, leading to the rich variety of ground and metastable magnetic states.

Recently, there have been a number of reports on metastable SkX formation done by quenching the sample under an applied magnetic field [25-32]. By utilizing this technique, a robust skyrmion state can be obtained in a thin plate of FeGe at low temperatures even at zero magnetic field, providing various opportunities for investigations of SkX stability under oblique, perpendicular, and negative magnetic fields. In this paper, we report on a resonant small-angle $\mathrm{X}$-ray scattering (RSXS) study of the polymorphic magnetic states in a thin strained FeGe lamella. Experiment reveals that the combination of the in-plane magnetic field and the tensile

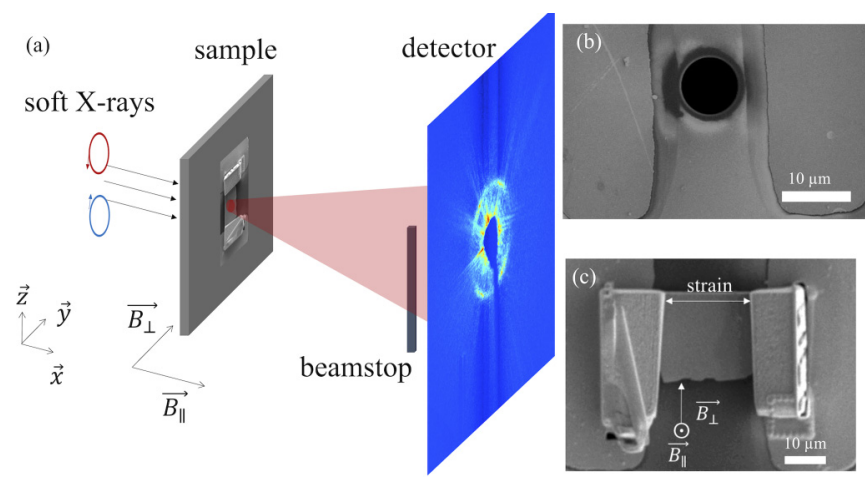

FIG. 2. (a) Schematic of the RSXS experiment. SEM image of the (b) sample aperture and (c) thin plate of FeGe fixed onto the membrane. Note that in the actual experiment, the detector plane and the beamstop were rotated by $-45^{\circ}$ to the $z$ axis in the $(y z)$ plane and magnetic field $B_{\perp}$ was applied by rotating the sample plane around the $z$ axis.

strain stabilizes the CSL with the $q$-vector perpendicular to the strain direction. Furthermore, we demonstrate that depending on the magnetic field direction and cooling protocol, the hysteretic transformations and the coexistence of metastable modulated states, such as SkX and CSL, are observed at low temperatures.

\section{EXPERIMENT}

The RSXS experiments were carried out at the soft-x-ray beamlines BL-16A, KEK Photon Factory (Tsukuba, Japan), and SEXTANTS, SOLEIL (Gif-sur-Yvette, France). A sketch of the experimental geometry at BL-16A and SEM images of the sample are given in Fig. 2. The experiments were performed with the circularly polarized soft x-rays of a photon energy $E=707 \mathrm{eV}$ corresponding to the $L_{3}$ absorption edge of Fe. The small-angle scattering setup at BL-16A was equipped with a high-vacuum chamber with a background pressure of $10^{-8}$ Torr [33]. The intensities of scattered beams were collected by an in-vacuum charge coupled device (CCD) detector of $512 \times 512$ pixels (Princeton Instruments, Trenton, NJ, USA) protected from the direct beam by a tungsten beamstop. A pair of Helmholtz coils provided a static magnetic field $B_{\|}$in the direction parallel to the incident beam. A magnetic field $B_{\perp}$ parallel to the sample plane was applied by rotating the sample by $90^{\circ}$ [i.e., around the $z$ axis in Fig. 2(a)]. Therefore, the RSXS patterns in the present study were measured at $B_{\perp}=0$ after ex situ application of $B_{\perp}$. The magnitude of a magnetic field provided by the electromagnet was in the range from 0 to $400 \mathrm{mT}$. The sample temperature was controlled by a He-flow-type refrigerator. The RESOXS setup at SEXTANTS was equipped with a quadruple magnet that allows the in situ observation of the scattering patterns for both in-plane and out-of-plane magnetic-field geometries in the field range from 0 to $150 \mathrm{mT}$ [34]. The data measured using the RESOXS setup are given in Appendix A.

The single-crystal sample of FeGe was grown by the chemical vapor transport method [35]. The lamella with a thickness of $150 \mathrm{~nm}$ was prepared by focused ion beam (FIB) milling and fixed to a gold-coated silicon nitride membrane behind 

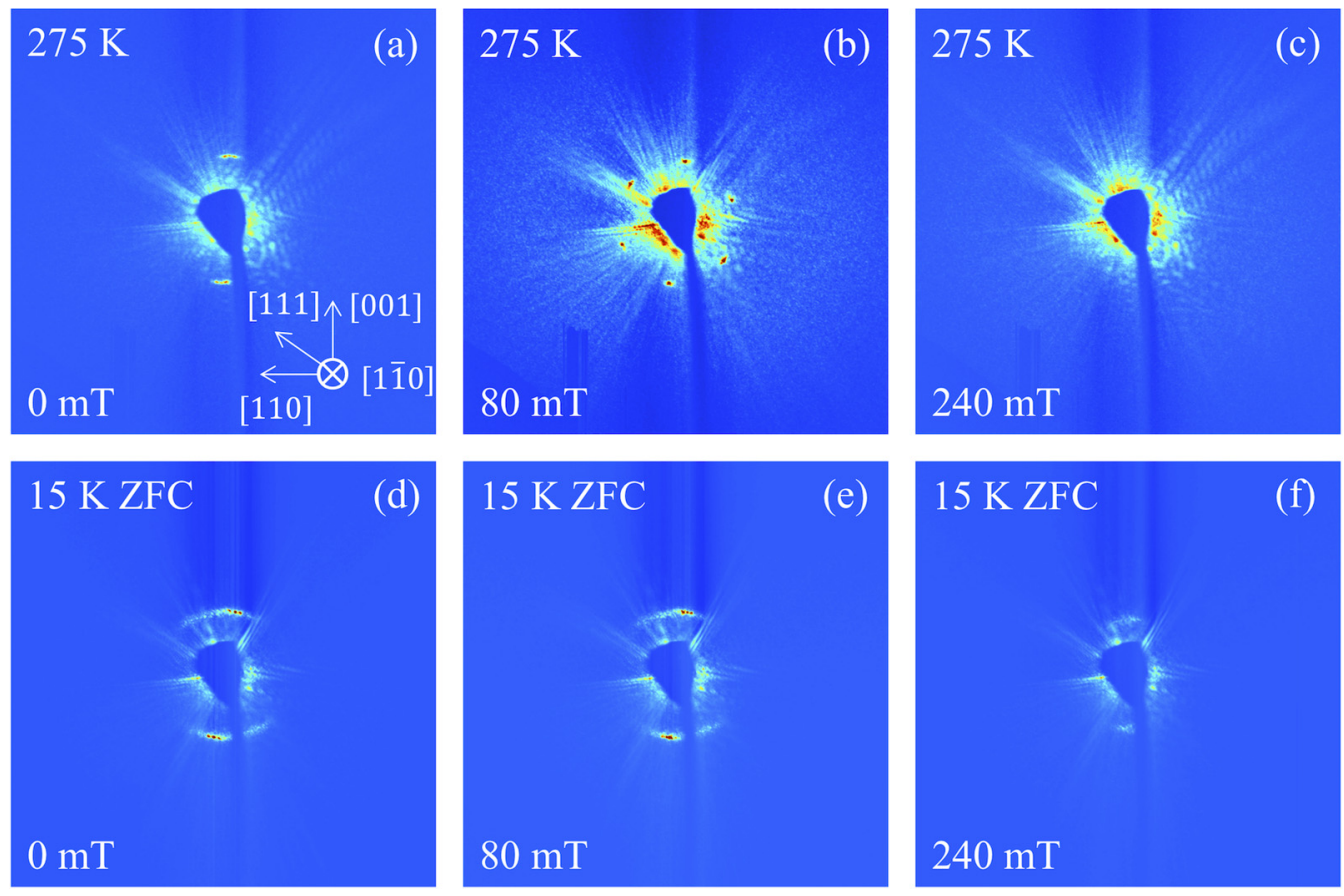

FIG. 3. Typical RSXS patterns for a strained FeGe sample. (a)-(c) At $T=275 \mathrm{~K}$ the helical state shown in (a) transforms to (b) SkX at 50-140 $\mathrm{mT}$ and (c) the conical/induced ferromagnetic state at $150 \mathrm{mT}$. (d)-(f) After zero-field cooling down to $T=15 \mathrm{~K}$ the helical state shown in (d) gradually transforms to the conical/induced ferromagnetic state with increasing magnetic field.

the aperture with a diameter of $8 \mu \mathrm{m}$ [Fig. 2(b)]. Tensile strain occurred due to the tungsten deposition at both sides of the thin plate and the difference of the thermal expansion coefficients between $\mathrm{FeGe}$ and $\mathrm{Si}_{3} \mathrm{~N}_{4}$ membrane [17,33]. The strain direction is shown in Fig. 2(c).

\section{RESULTS}

At $275 \mathrm{~K}$ the typical RSXS patterns arising from the helical, SkX, and conical/field-polarized phases of FeGe [33,3638] were observed [Figs. 3(a)-3(c)]. The orientation of the helical $q$-vector was pinned along the [001], while the orientation of the SkX was not fixed and the skyrmion lattice stochastically transformed between the single-domain and the multidomain states, whereas the relative rotation angle between domains was approximately $15^{\circ}$. This rotation indicates that in the present sample, the magnitude of the tensile strain at $T=275 \mathrm{~K}$ was not sufficient to lock the orientation of the SkX as it was observed in Refs. [16,17,33,39]. The dependence of magnetic scattering on magnetic field $B_{\|}$was measured at the lowest temperature $T=15 \mathrm{~K}$ after the zerofield-cooling (ZFC) procedure [Figs. 3(d)-3(f)]. ZFC led to the transition from the single-domain helical to the conical or field-polarized magnetic structure as the field increased from 0 to $150 \mathrm{mT}$ or above. No higher-order harmonics of the magnetic scattering were observed, indicating a direct helical-to-conical transition without the appearance of the intermediate CSL or SkX states. By increasing the magnetic field $B_{\|}$to the maximal value of $400 \mathrm{mT}$ and going back to zero field, two helical domains with the propagation vectors encompassed along the [111]-equivalent directions (Fig. 9 in Appendix B) appear. The intensity of the Bragg peak arising from the helical domain with the $q$-vector parallel to the strain is approximately five times stronger than the one with the perpendicular propagation direction. This indicates the enhanced stability of the magnetic helices with the $q$-vector along the tensile strain. Therefore, we assume the easy-plane anisotropy induced by the deformation with the anisotropy axis parallel to the strain.

Surprisingly, application and consequent withdrawal of the magnetic field $B_{\perp}=120 \mathrm{mT}$ perpendicular to the tensile strain in the sample plane leads to the appearance of secondorder Bragg reflections [Fig. 4(a)], which unambiguously indicates an anharmonicity of the magnetic modulation. Similar higher-order scattering peaks have been previously observed in other chiral magnets $[17,28,40]$ and centrosymmetric systems with long-periodic magnetic texture [41-43], and they can be applied as indicators of a spiral distortion. Therefore, the formation of a distorted helical texture, or CSL, was assisted by the magnetic field applied perpendicular to the strain in the thin plate plane, which has not been reported previously for $\mathrm{FeGe}$ or $\mathrm{Cu}_{2} \mathrm{OSeO}_{3}$. Observation of the CSL at zero field indicates the metastable character of this state. By applying the field $B_{\|}$normal to the sample surface, the evolution of the amplitudes and positions of the first-order (1q) and second-order $(2 q)$ magnetic Bragg peaks was recorded (Fig. 4). Interestingly, the CSL was not destroyed by the magnetic field, indicating rotation of the nonzero in-plane net magnetization component normal to the plane. We note that in the case of helical reorientation toward the applied $B_{\|}$field, the diffraction intensity would gradually decrease without manifestation of the higher harmonics. Therefore, instead of the usual helical-to-conical transition, we assume that the conical state competes with the metastable CSL when 

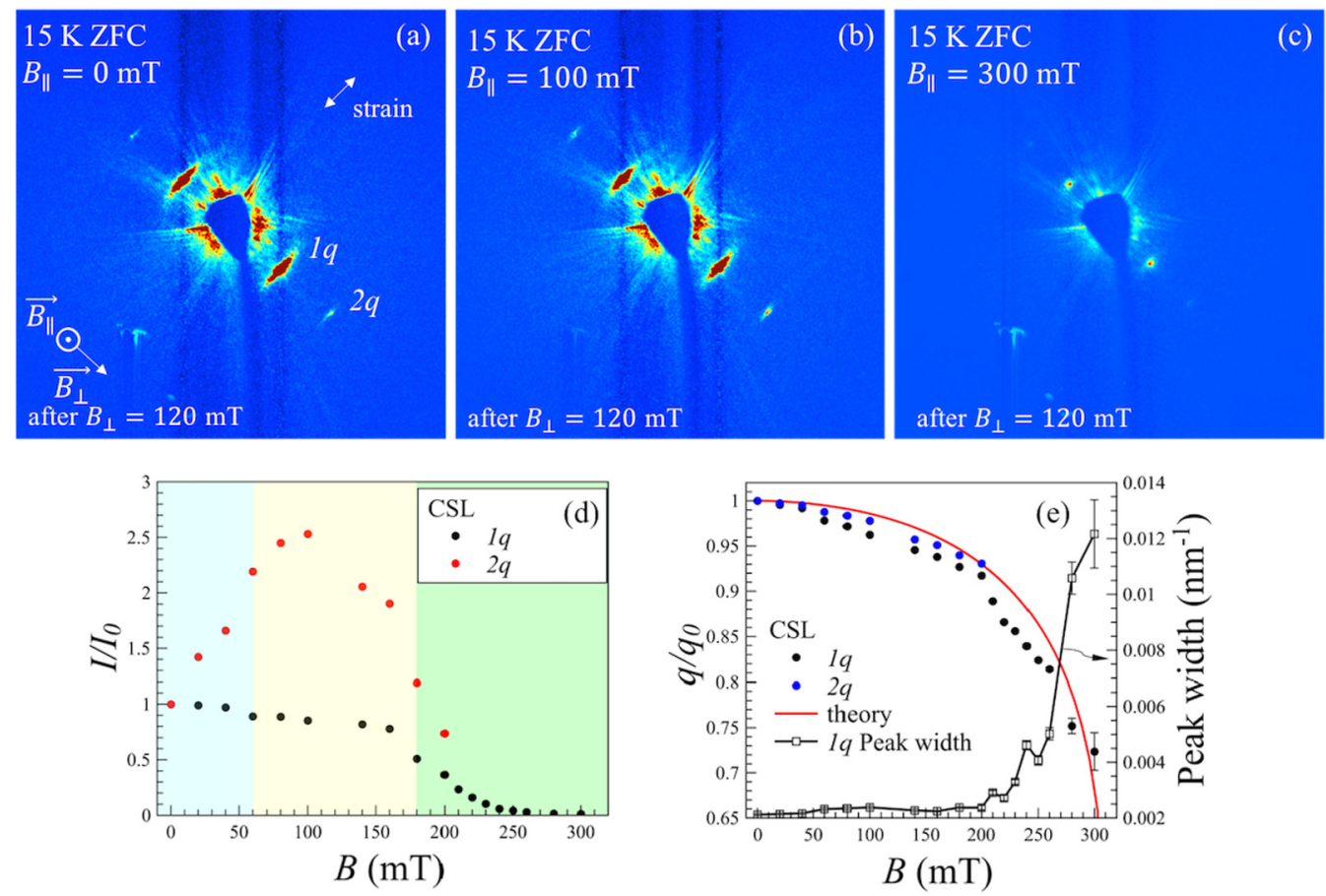

FIG. 4. RSXS patterns of strained FeGe lamella measured at $T=15 \mathrm{~K}$ after application and removal of $B_{\perp}=120 \mathrm{mT}$ at (a) $B_{\|}=0 \mathrm{mT}$, (b) $B_{\|}=100 \mathrm{mT}$, and (c) $B_{\|}=300 \mathrm{mT}$. First- and second-order Bragg peaks arising from CSL are indicated as $1 q$ and $2 q$, respectively. (d) Dependence of the relative intensity of the $1 q$ and $2 q$ peaks on applied magnetic field $B_{\|}$. (e) Dependence of the relative $q$-vector magnitude and FWHM of the $1 q$ and $2 q$ peaks on applied magnetic field $B_{\|}$.

the field $B_{\|}$is applied sequentially after $B_{\perp}$. When the field is increased above $200 \mathrm{mT}$, the CSL transforms to the conical state with the $q$-vector oriented parallel to the field direction. This field-induced transition from the in-plane helical to the out-of-plane conical state is mediated by the in-plane CSL, which is different from, for example, uniaxial helimagnet $\mathrm{CrNb}_{3} \mathrm{~S}_{6}$ where the conical phase is suppressed and a helicalto-field-polarized transition takes place via CSL phase [10]. Due to the gradual variation of the intensity, the magnitude of the $q$-vector, and the full width at half-maximum (FWHM) of the second-order Bragg peaks [Figs. 4(d) and 4(e)], we suggest there is a phase coexistence between the CSL and conical domains between 100 and $250 \mathrm{mT}$. The magnetic-field dependence of the $q$-vector in this process consists of the chiral sine-Gordon model [4,5] [Fig. 4(e)] for the lower field range $(0<B<100 \mathrm{mT})$. Notably, an in-plane field applied parallel to the strain axis does not induce the CSL, but it leads to the gradual rotation of the propagation vector toward the field (see Appendix A). A more accurate description of the CSL flop above $100 \mathrm{mT}$ is given in Sec. IV.

Next we explore the field-tuning between a metastable SkX state and CSL states. Field cooling (FC) from 275 to $15 \mathrm{~K}$ in an applied magnetic field $B_{\|}=120 \mathrm{mT}$ with a cooling rate of $0.1-0.4 \mathrm{~K} / \mathrm{s}$ led to the appearance of the metastable SkX, which remained even in zero field [Fig. 5(a)]. The magnetic contribution to RSXS dramatically increased at the low-temperature conditions, providing access to the higherorder Bragg peaks from SkX. The stability of the supercooled SkX over a perpendicular magnetic field has been studied with the protocol described in Sec. II. The evolution of the magnetic scattering intensity with $B_{\perp}$ is shown in Fig. 5. In zero field the SkX coexists with the helical domain [Fig. 5(a)]. After application of the in-plane magnetic field in a range from 20 to $200 \mathrm{mT}$, the magnetic texture first evolves into the coexisting SkX and CSL oriented along $B_{\perp}$ [Figs. 5(b)-5(d)], as is suggested by the sixfold pattern arising from SkX and $1 q$ and $2 q$ Bragg peaks from CSL. Domination of the metastable CSL over the supercooled SkX proceeds when increasing the in-plane field, and it also lowers the coherence of the SkX, as is indicated by the vanishing of the higher-order Bragg peaks of SkX [Fig. 5(d)]. After application of a field $B_{\perp}=$ $220 \mathrm{mT}$, a ringlike pattern coexistent with the diffraction spots from the CSL was observed. This pattern is attributed to the disordered skyrmion lattice or helical domains. The dependencies of the integrated intensity and magnitude of the $q$-vector of the first-order Bragg peaks of SkX and CSL are summarized in Figs. 6(a) and 6(b). The low- and intermediatefield regions $\left(0<B_{\perp}<200 \mathrm{mT}\right)$ correspond to the coexisting SkX and helical/CSL magnetic textures. Next, the intensity dependence in the higher-field region $\left(200<B_{\perp}<300 \mathrm{mT}\right)$ demonstrates mixed CSL and disordered skyrmion phases, although the scattering intensity from the SkX is vanishing compared to the single- $q$ modulation [Fig. 6(a)]. Surprisingly, the $q$-vector length of the skyrmion texture tends to increase with the increment of the magnetic field [Fig. 6(b)], which is opposite to the tendency for the SkX, isolated skyrmions, or in-plane skyrmion tubes in the conical/ferromagnetic background [44-46]. By contrast, application of the in-plane field does not influence the magnitude of the CSL wave vector [Fig. 6(b)]. Application of the in-plane field above $300 \mathrm{mT}$ 

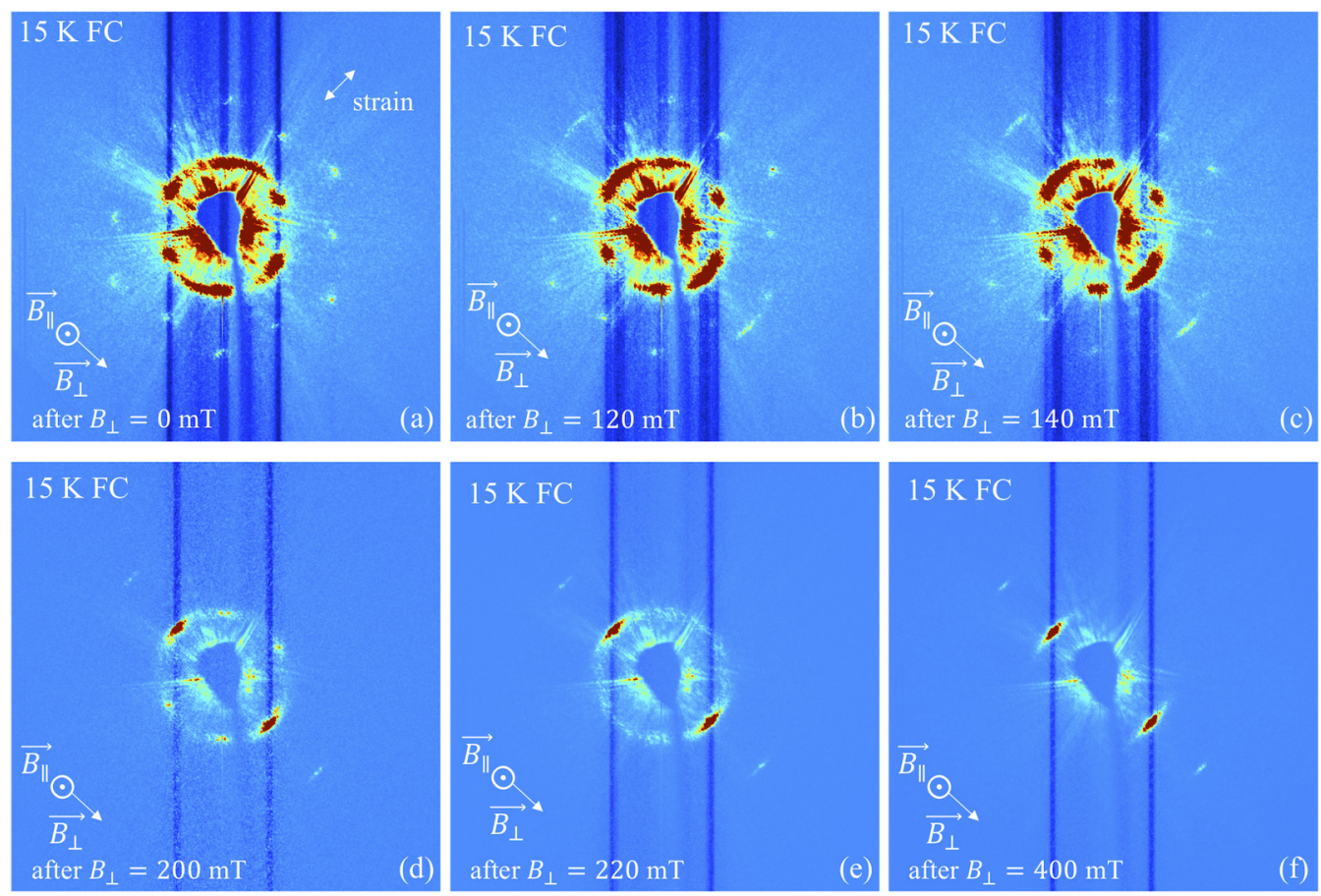

FIG. 5. RSXS patterns for strained FeGe lamella measured at $T=15 \mathrm{~K}$ and zero-field condition after field cooling in $B_{\|}=120 \mathrm{mT}$ and applied in-plane magnetic field (a) $B_{\perp}=0 \mathrm{mT}$, (b) $B_{\perp}=120 \mathrm{mT}$, (c) $B_{\perp}=140 \mathrm{mT}$, (d) $B_{\perp}=200 \mathrm{mT}$, (e) $B_{\perp}=220 \mathrm{mT}$, and (f) $B_{\perp}=400 \mathrm{mT}$.

completely destroys the skyrmion crystal, and only the CSL remains.

\section{DISCUSSIONS}

Recently, Lorentz transmission electron microscopy (LTEM) experiments showing a thickness-dependent stability of helicoidal, conical, and skyrmion states in FeGe lamella with a thickness gradient in the presence of a magnetic field have been discussed in Ref. [47] in the context of chiral surface twists. In the present section, we focus on a theoretical model that is sufficient to describe the experimental RSXS data obtained for the zero-field-cooling case, i.e., considering only helical, conical, and CSL phases. To discuss the experimental data obtained for the metastable SkX, a micromagnetic simulation has been performed.

\section{A. Chiral soliton lattice}

Chiral soliton lattices are usually attributed to uniaxial helimagnets, where a spiral can propagate only along a single direction, and spins are collinear within the planes that are perpendicular to the axis. In this subsection, we recall the classical solutions for uniaxial helimagnets in an external in-plane magnetic field or with additional in-plane easy-axis anisotropy (provided, e.g., by tensile stress), which lead to the proper screw distortion.

Here we consider a simple model of a uniaxial helimagnet on a tetragonal lattice, assuming an isotropic ferromagnetic exchange that is the same in all directions, and Dzyaloshinskii-Moriya interaction along the $z$-axis between nearest neighbors in neighboring $x y$-planes. Additionally, we introduce to the model an in-plane magnetic field and an inplane easy-axis anisotropy. The corresponding system Hamiltonian reads

$$
\begin{aligned}
\mathcal{H}= & -J \sum_{\langle i j\rangle} \mathbf{S}_{i} \cdot \mathbf{S}_{j}-\sum_{[i j]} \mathbf{D} \cdot\left[\mathbf{S}_{i} \times \mathbf{S}_{j}\right] \\
& -\sum_{i} g \mu_{B} \mathbf{H} \cdot \mathbf{S}_{i}-A \sum_{i} \mathbf{S}_{x, i}^{2} .
\end{aligned}
$$

Henceforth, we consider the magnetic field in energy units, $h=g \mu_{B} H$. In the absence of anisotropy and external field, the ground state of this system is the spin helix propagating along the $z$-axis with spins rotating in the $x y$-plane and modulation vector $\mathbf{q}=(0,0, D / J)$. When $q \ll 1$ one can rewrite Hamiltonian (1) in a continuous form using the variable $\varphi(z)$, which corresponds to a spin polar angle in the $x y$-plane.

For the system in an external effective in-plane magnetic field $\alpha=h / S J$, the solution is given by the following expressions [5]:

$$
\begin{gathered}
\varphi(z)=2 \operatorname{am}\left(\sqrt{\frac{\alpha}{m}} z, m\right), \\
L_{\text {kink }}=\frac{8 K(m) E(m)}{\pi q}, \\
\frac{4 E(m)}{\pi q}=\sqrt{\frac{m}{\alpha}} .
\end{gathered}
$$

Here $m$ is the parameter, which should be found from the preceding equation, $K(m)$ and $E(m)$ are the complete elliptic integrals of the first and second kinds, respectively, and am 

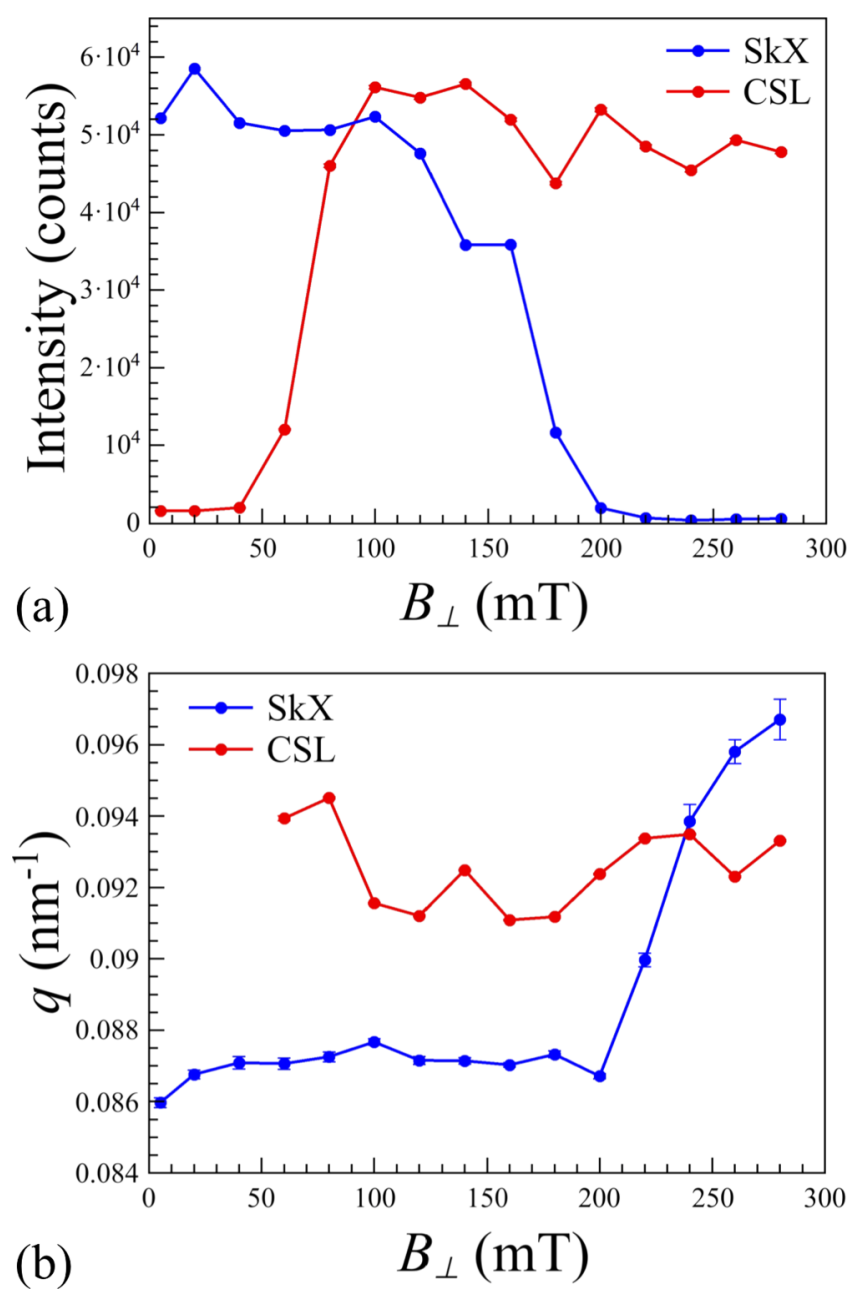

FIG. 6. In-plane magnetic-field dependence of (a) the intensity and (b) the $q$-vector magnitude of the Bragg peaks corresponding to the skyrmion lattice (SkX) and the chiral soliton lattice (CSL).

is the Jacobi amplitude function. These equations yield the periodicity of magnetic modulation $L_{\text {kink }}=2 \pi / q$ for the zero external field $(\alpha=0, m=0)$. The phase transition to the fully polarized state takes place at $m=1$, for which $\alpha_{c}=(\pi q / 4)^{2}$. At small magnetic fields $\alpha \ll \alpha_{c}$ we find $m=4 \alpha / q^{2}(1-$ $\left.2 \alpha / q^{2}\right)$, and the corresponding expression for the system energy per one spin is given by

$$
\frac{\varepsilon}{N}=-3 S^{2} J-\frac{S^{2} D^{2}}{2 J}-\frac{J h^{2}}{4 D^{2}} .
$$

In the case of nonzero in-plane easy-axis anisotropy $(A \neq$ 0 ), the model can be directly mapped onto the previous case with $\tilde{q}=2 q$ and $\tilde{\alpha}=2 A / J$. Considering anisotropy as a small perturbation $\left(A \ll D^{2} / J\right)$, we can derive the energy of the corresponding magnetic structure per one spin in the following form:

$$
\frac{\varepsilon}{N}=-3 S^{2} J-\frac{S^{2} D^{2}}{2 J}-\frac{S^{2} A}{2}-\frac{S^{2} J A^{2}}{4 D^{2}} .
$$

Now we consider an external magnetic field along the $z$-direction. Assuming that all spins are canted to the same angle $\theta$ toward the field direction, after the minimization of energy per one spin over $\theta$, we obtain

$$
\frac{\varepsilon}{N}=-3 S^{2} J-\frac{S^{2}}{2}\left(\frac{D^{2}}{J}+A+\frac{J A^{2}}{2 D^{2}}\right)-\frac{h^{2}}{2\left[\frac{D^{2}}{J}+A+\frac{J A^{2}}{2 D^{2}}\right]} .
$$

In this form, this model can be applied to analyze the cubic helimagnets with tensile strain along one of the directions.

\section{B. Spiral plane flop}

In this subsection, we study a simple model related to strained B20 helimagnets. It allows us to describe the spiral plane flop observed experimentally (Fig. 4). In this case, we consider only nearest-neighbor exchange interaction and DMI with vectors along the corresponding bonds. The corresponding model Hamiltonian reads

$$
\begin{aligned}
\mathcal{H} & =\mathcal{H}_{\mathrm{ex}}+\mathcal{H}_{\mathrm{dm}}+\mathcal{H}_{\mathrm{an}}+\mathcal{H}_{Z}, \\
\mathcal{H}_{\mathrm{ex}} & =-\sum_{\langle i j\rangle} J_{i j} \mathbf{S}_{i} \cdot \mathbf{S}_{i}, \\
\mathcal{H}_{\mathrm{dm}} & =-\sum_{\langle i j\rangle} \mathbf{D}_{i j} \cdot\left[\mathbf{S}_{i} \times \mathbf{S}_{j}\right], \\
\mathcal{H}_{\mathrm{an}} & =-A \sum_{i}\left(S_{x, i}^{2}+S_{y, i}^{2}\right), \\
\mathcal{H}_{Z} & =-\sum_{i} \mathbf{h} \cdot \mathbf{S}_{i} .
\end{aligned}
$$

Here we consider the B20 cubic helimagnet with tensile strain along the $z$-axis. This yields the $x y$ easy-plane and the difference between exchange and DMI for in-plane bonds and along the $z$-axis, namely $J, D$ and $J^{\prime}, D^{\prime}$.

If we apply a magnetic field along the $x$-axis, two competing spin textures can arise: CSL of $\mathbf{q}=(0,0, q)$ with spins in the $x y$-plane, and a bunched cone of $\mathbf{q}=(q, 0,0)$ with spin rotating in the $y z$-plane. The former is energetically favorable at small magnetic fields, while the latter becomes stable as the field increases due to spin canting toward the magnetic-field direction. Analytically this stems from a competition between two energies:

$$
\begin{aligned}
\frac{\varepsilon_{x y}}{N}= & -2 S^{2} J-S^{2} J^{\prime}-\frac{S^{2} D^{\prime 2}}{2 J^{\prime}}-S^{2} A-\frac{J^{\prime} h^{2}}{4 D^{\prime 2}} \\
\frac{\varepsilon_{y z}}{N}= & -2 S^{2} J-S^{2} J^{\prime}-\frac{S^{2} D^{2}}{2 J}-\frac{S^{2} A}{2}-\frac{J S^{2} A^{2}}{4 D^{2}} \\
& -\frac{h^{2}}{2\left[A+D^{2} / J+J A^{2} /\left(2 D^{2}\right)\right]} .
\end{aligned}
$$

For clarity we consider a specific situation. Let $D^{2} / J=$ $D^{\prime 2} / J^{\prime}$, and the anisotropy constant $A \ll D^{2} / J$ is small. It is obvious from Eqs. (8) and (9) that the energy of the bunched cone texture decreases in magnetic field twice as fast as the CSL one, and at a sufficiently large external field a spiral 


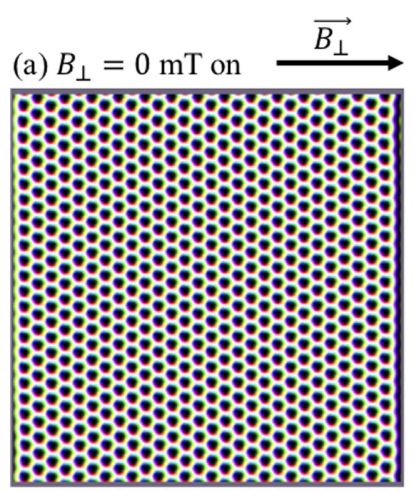

(b) $B_{\perp}=0 \mathrm{mT}$ off

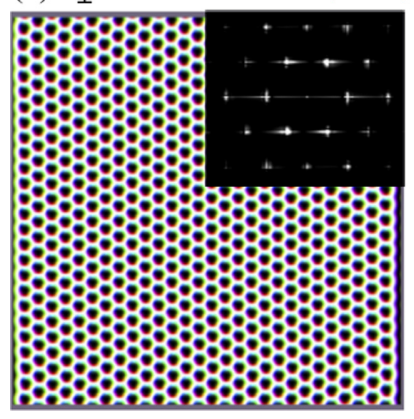

(c) $B_{\perp}=380 \mathrm{mT}$ on

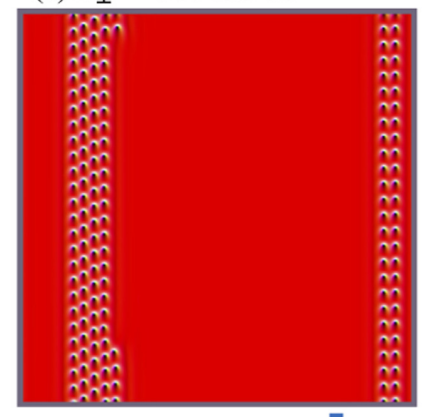

(d) $B_{\perp}=380 \mathrm{mT}$ off

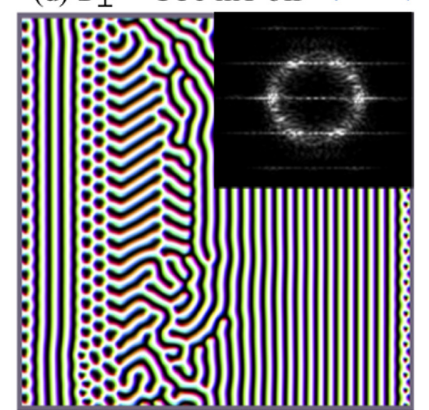

(e) $B_{\perp}=400 \mathrm{mT}$ on
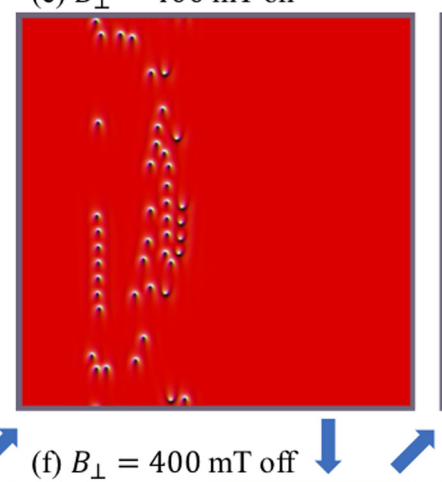

(g) $B_{\perp}=440 \mathrm{mT}$ on

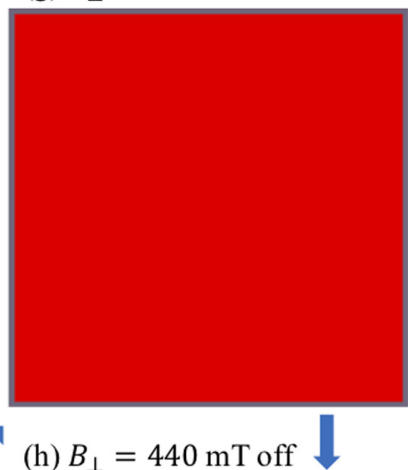

FIG. 7. Micromagnetic simulation of a FeGe lamella with uniaxial anisotropy after application and removal of in-plane magnetic fields (a,b) $B_{\perp}=0 \mathrm{mT}$, (c,d) $B_{\perp}=380 \mathrm{mT}$, (e,f) $B_{\perp}=400 \mathrm{mT}$, and (g,h) $B_{\perp}=440 \mathrm{mT}$. The sequence of the field application protocol is denoted by blue arrows. In-plane magnetic field was consequently increased with $20 \mathrm{mT}$ steps (" $B_{\perp}$ on" state) and switched to zero (" $B_{\perp}$ off" state). Insets show fast Fourier transform (FFT) images of the zero-field magnetization states after the corresponding in-plane field training for qualitative comparison with experimental RSXS patterns (Fig. 5).

plane flop takes place. The transition point is estimated by the equation

$$
\frac{A S^{2}}{2}=\frac{J h_{\text {flop }}^{2}}{4 D^{2}} .
$$

This gives

$$
h_{\text {flop }}=S \sqrt{\frac{2 A D^{2}}{J}} .
$$

We can easily confirm that $h_{\text {flop }} \ll h_{c} \propto S D^{2} / J$, which justifies the small-field approach for CSL energy. By using the measured ratio $h_{\text {flop }} / h_{c} \approx 1 / 3$ this equation leads to the estimation of the induced easy-plane anisotropy constant $A \approx$ $1.6 \times 10^{4} \mathrm{~J} / \mathrm{m}^{3}$.

\section{Micromagnetic simulation of the skyrmion lattice in the in-plane magnetic field}

By assuming the easy-plane anisotropy axis along the deformation direction and magnitude $A=1.6 \times 10^{4} \mathrm{~J} / \mathrm{m}^{3}$ estimated from the analytical theory and experimental results, we performed the simulation of the skyrmion lattice in the strained FeGe lamella by using the MUMAX ${ }^{3}$ package [48]. In the simulation, we used a two-dimensional thin plate $2048 \times 2048 \mathrm{~nm}^{2}$ and with a thickness of 100 $\mathrm{nm}$ with periodic boundary conditions along the in-plane directions. The parameters used for the simulation were obtained experimentally by microwave spin-wave spectroscopy in Ref. [49]. Metastable SkX was introduced as the initial zero-field state [Figs. 7(a) and 7(b)]. The in-plane magnetic field deforms the metastable skyrmions resulting in elongation toward the field direction. By increasing the magnitude of the magnetic field, aligned chains of skyrmions show up in the ferromagnetic background at $B_{\perp}=380-400 \mathrm{mT}$ [Figs. 7(c) and 7(e)]. Interestingly, when the field is released, both elongated and compressed skyrmion vortices of smaller diameter appear at the boundaries between the helical and skyrmion chain domains [Fig. 7(d)]. Finally, by applying a field of $B_{\perp}=440 \mathrm{mT}$, the sample undergoes a transition to the homogeneously magnetized state [Fig. $7(\mathrm{~g})]$. The procedure of consequent application/removal of the in-plane magnetic field reproduces the experimental conditions described in the previous sections: the in-plane magnetic field $B_{\perp}$ was consequently applied to the initial zero-field $\mathrm{SkX}$ state in 20-mT increments and switched off. By using this protocol, the micromagnetic simulation delivers skyrmion clusters and individual skyrmions coexisting with the helical domains with the $q$-vector oriented along the in-plane field direction [Figs. 7(d) and 7(f)]. For comparison with experimental RSXS data, the corresponding fast Fourier transform (FFT) patterns were calculated for the out-of-plane magnetization component of the resultant zero-field states [insets in Figs. 7(b), 7(d) 7(h), and $7(\mathrm{f})]$.

We suppose that the ringlike RSXS patterns observed in the experiment [Figs. 5(c) and 5(f)] may correspond to the mixed skyrmion clusters and multidomain helical [mazelike pattern 
in Figs. 7(d), 7(h) and 7(f)] states delivered by the micromagnetic simulation. In our case, the mixed skyrmion clusters and disordered helical pitches coexist with the metastable CSL discussed in the previous sections. Although the simulation does not allow us to reproduce the specific incremental $q$-dependence on the magnetic field, the attractive nature of the skyrmion clusters in the helical background has already been observed by LTEM [50]. The skyrmion condensation is assisted by the nonaxisymmetric skyrmion shapes induced by the in-plane magnetic field [51]. The specific compressed skyrmions formed at the domain boundary between the skyrmion cluster and the helical domain hint at the possible scenario of the $q$-vector increase observed in the RSXS experiment [Fig. 6(b)]. Contributions of more exotic three-dimensional spin textures, such as stacked spirals [52], chiral bobbers [53-55], quasimonopoles [56], and in-plane skyrmion tubes $[46,57,58]$, to the scattering patterns are another possible explanation of this result. Further real-space investigations of the possible three-dimensional states using cryogenic coherent [36,59] or focused [46] soft-x-ray methods will help to clarify this question.

\section{CONCLUSION}

We have investigated the low-temperature magnetic states in a strained lamella of $\mathrm{FeGe}$ by means of resonant smallangle soft-x-ray scattering. If the magnetic field is applied in the plane of lamella and perpendicular to the tensile strain direction at $15 \mathrm{~K}$, the chiral soliton lattice shows up as a metastable state when the field is removed. The skyrmion lattice is stabilized by the field cooling and survives even at zero field at low temperatures. Further application of the in-plane magnetic field results in the coexistence of the metastable skyrmion and chiral soliton lattices. The skyrmion crystal unwinds to the helicoidal state passing through the intermediate phase consisting of the isolated skyrmion clusters and disordered helices. This polymorphism of the lowtemperature zero-field magnetic states results from the complex landscape of magnetic interactions, including exchange, anisotropy, and Dzyaloshinskii-Moriya interactions. Finally, our measurements have shown a nontrivial interaction between the coexisting metastable skyrmion and chiral soliton lattices, namely unexpected attractive behavior in skyrmion clusters. The revealed effect of the tensile strain can be poten- tially employed to control skyrmion and chiral soliton lattices in helimagnetic materials. By modifying the anisotropy and Dzyaloshinskii-Moriya interaction in cubic chiral magnets, one can stabilize the rich variety of magnetic solitonic textures. It is also possible that the strain tuning can help to stabilize the skyrmion lattice in the chiral helimagnets with lower crystal symmetry.

\section{ACKNOWLEDGMENTS}

Soft-x-ray scattering experiments were performed at KEK Photon Factory as a part of Projects No. 2015S2-007 and No. 2018S2-006, and at synchrotron Soléil as part of Proposal No. 20181292. This research was supported in part by PRESTO Grant No. JPMJPR177A, by Grants-in-Aid for Scientific Research No. JP16H05990, No. JP19H04399, and No. JP20H05155 from the Japan Society for the Promotion of Science (JSPS), by MEXT QuantumLeap Flagship Program (MEXT Q-LEAP) Grant No. JPMXS0120184122, by Research Foundation for Opto-Science and Technology, by Materials research by Information Integration Initiative $\left(\mathrm{MI}^{2} \mathrm{I}\right)$ project of the Support Program for Starting Up Innovation Hub from JST, the Japan Society for the Promotion of Science through the Funding Program for World-Leading Innovative R\&D on Science and Technology (FIRST Program). V.U. acknowledges support from the SNF Sinergia CRSII5-171003 NanoSkyrmionics. The theoretical part of this study conducted by $\mathrm{O}$. Utesov was funded by RFBR according to the research project 18-32-00083. The research at synchrotron SOLEIL leading to this result has been supported by the project CALIPSOplus under Grant Agreement 730872 from the EU Framework Programme for Research and Innovation HORIZON 2020. The authors thank A. V. Syromyatnikov, Y. Okamura, and J. S. White for fruitful discussions, and Y. Yokoyama and L. Yu for technical assistance.

\section{APPENDIX A: CONICAL MODULATION FOR THE IN-PLANE FIELD || STRAIN}

The quadrupole magnet of the RESOXS setup allowed the in situ measurement of the magnetic texture evolution for the $B_{\perp} \|$ strain geometry (Fig. 8). The upper and the left parts of the detector were shadowed by the magnet and the beamstop, respectively.
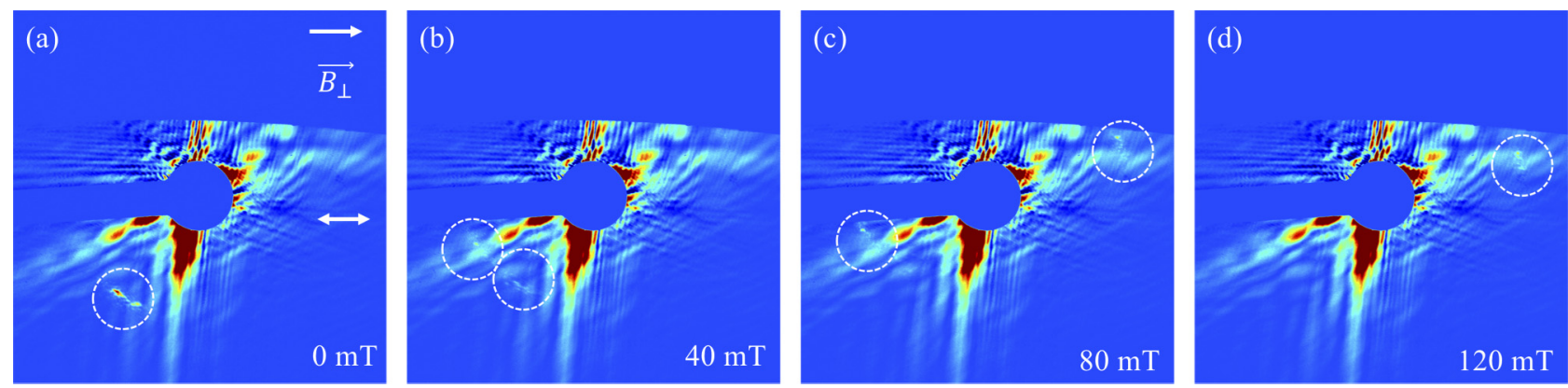

FIG. 8. RSXS patterns for strained FeGe lamella measured at $T=32 \mathrm{~K}$ after zero-field cooling and in-plane field (a) $B_{\perp}=0 \mathrm{mT}$, (b) $B_{\perp}=$ $40 \mathrm{mT}$, (c) $B_{\perp}=80 \mathrm{mT}$, and (d) $B_{\perp}=120 \mathrm{mT}$ applied parallel to the tensile strain direction. Magnetic Bragg peaks are highlighted by the dashed circles. 


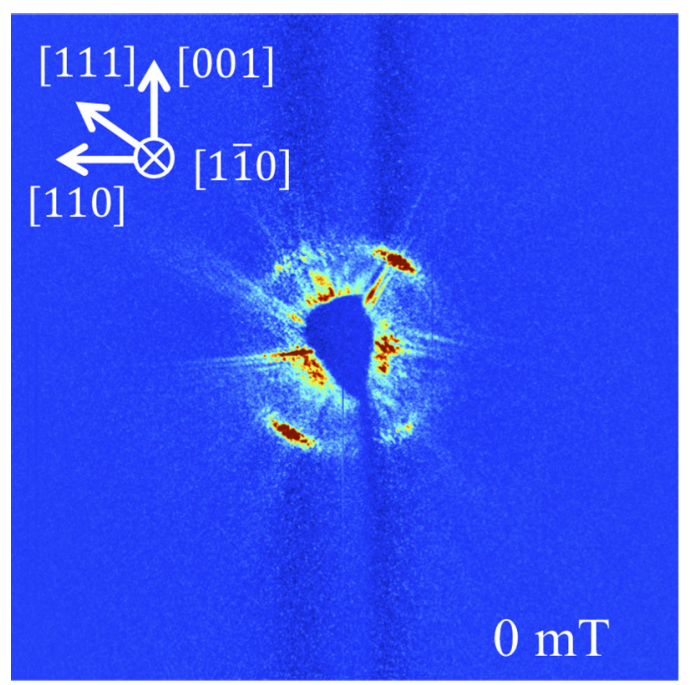

FIG. 9. RSXS pattern for strained FeGe lamella measured at $T=15 \mathrm{~K}$ and zero-field condition after zero-field cooling and outof-plane field training at $B_{\|}=400 \mathrm{mT}$.

The sample was oriented in the beam just as the strain axis was in the horizontal plane and parallel to the magnetic field [Fig. 8(a)]. The RSXS patterns as a function of applied field were measured after ZFC from room temperature to the lowest available temperature $T=32 \mathrm{~K}$. The propagation direction of the helical texture after ZFC is similar to the measurements at $T=275$ and $15 \mathrm{~K}$ (Fig. 3). First, at a magnetic field of $B_{\perp}=$ $40 \mathrm{mT}$ applied parallel to the tensile strain, the helical state splits into two domains [Fig. 8(b)]. Next, the two domains merge and the whole texture gradually rotates toward the field direction [Figs. 8(c) and 8(d)]. Notably, no higher-harmonic scattering can be observed in the scattering patterns, confirming the smooth helical-to-conical transformation without an intermediate CSL state. This observation is in excellent agreement with our theoretical model. The field-polarized state was not achieved due to the limited amplitude of the magnetic field in the present setup.

\section{APPENDIX B: MULTIDOMAIN HELICAL STATE AFTER ZERO-FIELD COOLING AND OUT-OF-PLANE FIELD TRAINING}

Zero-field cooling from room temperature to $T=15 \mathrm{~K}$ and subsequent out-of-plane field training at $B_{\|}=400 \mathrm{mT}$ result in the multidomain helical state (Fig. 9). The propagation vectors of the helical modulation are roughly aligned with [111]-equivalent crystallographic axes.
[1] I. Dzyaloshinsky, J. Phys. Chem. Solids 4, 241 (1958).

[2] T. Moriya, Phys. Rev. 120, 91 (1960).

[3] P. Bak and M. H. Jensen, J. Phys. C 13, L881 (1980).

[4] I. Dzyaloshinskii, Sov. Phys. JETP 20 (1965).

[5] Y. A. Izyumov, Phys. Usp. 27, 845 (1984).

[6] I. G. Bostrem, J. I. Kishine, and A. S. Ovchinnikov, Phys. Rev. B 78, 064425 (2008).

[7] K. Koumpouras, A. Bergman, O. Eriksson, and D. Yudin, Sci. Rep. 6, 25685 (2016).

[8] Y. Togawa, Y. Kousaka, K. Inoue, and J.-i. Kishine, J. Phys. Soc. Jpn. 85, 112001 (2016).

[9] F. J. T. Goncalves, T. Sogo, Y. Shimamoto, Y. Kousaka, J. Akimitsu, S. Nishihara, K. Inoue, D. Yoshizawa, M. Hagiwara, M. Mito, R. L. Stamps, I. G. Bostrem, V. E. Sinitsyn, A. S. Ovchinnikov, J. Kishine, and Y. Togawa, Phys. Rev. B 95, 104415 (2017).

[10] Y. Togawa, T. Koyama, K. Takayanagi, S. Mori, Y. Kousaka, J. Akimitsu, S. Nishihara, K. Inoue, A. Ovchinnikov, and J.-i. Kishine, Phys. Rev. Lett. 108, 107202 (2012).

[11] Y. Togawa, Y. Kousaka, S. Nishihara, K. Inoue, J. Akimitsu, A. S. Ovchinnikov, and J. Kishine, Phys. Rev. Lett. 111, 197204 (2013).

[12] J.-I. Yonemura, Y. Shimamoto, T. Kida, D. Yoshizawa, Y. Kousaka, S. Nishihara, F. J. T. Goncalves, J. Akimitsu, K. Inoue, M. Hagiwara, and Y. Togawa, Phys. Rev. B 96, 184423 (2017).

[13] C. Tabata, Y. Yamasaki, Y. Yokoyama, R. Takagi, T. Honda, Y. Kousaka, J. Akimitsu, and H. Nakao (unpublished).

[14] S. Mühlbauer, B. Binz, F. Jonietz, C. Pfleiderer, A. Rosch, A. Neubauer, R. Georgii, and P. Böni, Science 323, 915 (2009).
[15] X. Z. Yu, Y. Onose, N. Kanazawa, J. Park, J. Han, Y. Matsui, N. Nagaosa, and Y. Tokura, Nature (London) 465, 901 (2010).

[16] K. Shibata, J. Iwasaki, N. Kanazawa, S. Aizawa, T. Tanigaki, M. Shirai, T. Nakajima, M. Kubota, M. Kawasaki, H. Park et al., Nat. Nanotechnol. 10, 589 (2015).

[17] Y. Okamura, Y. Yamasaki, D. Morikawa, T. Honda, V. Ukleev, H. Nakao, Y. Murakami, K. Shibata, F. Kagawa, S. Seki et al., Phys. Rev. B 96, 174417 (2017).

[18] A. S. Sukhanov, P. Vir, A. Heinemann, S. E. Nikitin, D. Kriegner, H. Borrmann, C. Shekhar, C. Felser, and D. S. Inosov, Phys. Rev. B 100, 180403(R) (2019).

[19] M. N. Wilson, E. A. Karhu, A. S. Quigley, U. K. Rößler, A. B. Butenko, A. N. Bogdanov, M. D. Robertson, and T. L. Monchesky, Phys. Rev. B 86, 144420 (2012).

[20] S. X. Huang and C. L. Chien, Phys. Rev. Lett. 108, 267201 (2012).

[21] Y. Li, N. Kanazawa, X. Z. Yu, A. Tsukazaki, M. Kawasaki, M. Ichikawa, X. Jin, F. Kagawa, and Y. Tokura, Phys. Rev. Lett. 110, 117202 (2013).

[22] N. A. Porter, C. S. Spencer, R. C. Temple, C. J. Kinane, T. R. Charlton, S. Langridge, and C. H. Marrows, Phys. Rev. B 92, 144402 (2015).

[23] N. Kanazawa, J. S. White, H. M. Rønnow, C. D. Dewhurst, Y. Fujishiro, A. Tsukazaki, Y. Kozuka, M. Kawasaki, M. Ichikawa, F. Kagawa, and Y. Tokura, Phys. Rev. B 94, 184432 (2016).

[24] S. Zhang, I. Stasinopoulos, T. Lancaster, F. Xiao, A. Bauer, F. Rucker, A. Baker, A. Figueroa, Z. Salman, F. Pratt et al., Sci. Rep. 7, 123 (2017).

[25] H. Oike, A. Kikkawa, N. Kanazawa, Y. Taguchi, M. Kawasaki, Y. Tokura, and F. Kagawa, Nat. Phys. 12, 62 (2016). 
[26] K. Karube, J. S. White, D. Morikawa, M. Bartkowiak, A. Kikkawa, Y. Tokunaga, T.-H. Arima, H. M. Rønnow, Y. Tokura, and Y. Taguchi, Phys. Rev. Mater. 1, 074405 (2017).

[27] X. Z. Yu, D. Morikawa, T. Yokouchi, K. Shibata, N. Kanazawa, F. Kagawa, T.-H. Arima, and Y. Tokura, Nat. Phys. 1 (2018).

[28] T. Nakajima, V. Ukleev, K. Ohishi, H. Oike, F. Kagawa, S.-I. Seki, K. Kakurai, Y. Tokura, and T.-H. Arima, J. Phys. Soc. Jpn. 87, 094709 (2018).

[29] F. Qian, L. J. Bannenberg, H. Wilhelm, G. Chaboussant, L. M. Debeer-Schmitt, M. P. Schmidt, A. Aqeel, T. T. Palstra, E. Brück, A. J. Lefering et al., Sci. Adv. 4, eaat7323 (2018).

[30] J. White, I. Živković, A. Kruchkov, M. Bartkowiak, A. Magrez, and H. Rønnow, Phys. Rev. Appl. 10, 014021 (2018).

[31] A. Chacon, L. Heinen, M. Halder, A. Bauer, W. Simeth, S. Mühlbauer, H. Berger, M. Garst, A. Rosch, and C. Pfleiderer, Nat. Phys. 14, 936 (2018).

[32] M. Wilson, M. Birch, A. Štefančič, A. Twitchett-Harrison, G. Balakrishnan, T. Hicken, R. Fan, P. Steadman, and P. Hatton, Phys. Rev. Res. 2, 013096 (2020).

[33] Y. Yamasaki, D. Morikawa, T. Honda, H. Nakao, Y. Murakami, N. Kanazawa, M. Kawasaki, T.-H. Arima, and Y. Tokura, Phys. Rev. B 92, 220421(R) (2015).

[34] N. Jaouen, J.-M. Tonnerre, G. Kapoujian, P. Taunier, J.-P. Roux, D. Raoux, and F. Sirotti, J. Synch. Radiat. 11, 353 (2004).

[35] N. Kanazawa, Magnetic and transport properties in b20-type germanides, in Charge and Heat Transport Phenomena in Electronic and Spin Structures in B20-type Compounds (Springer, Tokyo, 2015), pp. 29-44.

[36] V. Ukleev, Y. Yamasaki, D. Morikawa, N. Kanazawa, Y. Okamura, H. Nakao, Y. Tokura et al., Quantum Beam Sci. 2, 3 (2018).

[37] D. Burn, S. Zhang, S. Wang, H. Du, G. Van Der Laan, and T. Hesjedal, Phys. Rev. B 100, 184403 (2019).

[38] D. M. Burn, S. Wang, W. Wang, G. van der Laan, S. Zhang, H. Du, and T. Hesjedal, Phys. Rev. B 101, 014446 (2020).

[39] Y. Okamura, Y. Yamasaki, D. Morikawa, T. Honda, V. Ukleev, H. Nakao, Y. Murakami, K. Shibata, F. Kagawa, S. Seki et al., Phys. Rev. B 95, 184411 (2017).

[40] S. Grigoriev, S. Maleyev, A. Okorokov, Y. O. Chetverikov, P. Böni, R. Georgii, D. Lamago, H. Eckerlebe, and K. Pranzas, Phys. Rev. B 74, 214414 (2006).

[41] H. Dürr, E. Dudzik, S. Dhesi, J. Goedkoop, G. Van der Laan, M. Belakhovsky, C. Mocuta, A. Marty, and Y. Samson, Science 284, 2166 (1999).
[42] O. Hellwig, G. Denbeaux, J. Kortright, and E. E. Fullerton, Physica B 336, 136 (2003).

[43] R. D. Desautels, L. DeBeer-Schmitt, S. A. Montoya, J. A. Borchers, S.-G. Je, N. Tang, M.-Y. Im, M. R. Fitzsimmons, E. E. Fullerton, and D. A. Gilbert, Phys. Rev. Mater. 3, 104406 (2019).

[44] A. Bogdanov and A. Hubert, J. Magn. Magn. Mater. 138, 255 (1994).

[45] D. McGrouther, R. Lamb, M. Krajnak, S. McFadzean, S. McVitie, R. Stamps, A. Leonov, A. Bogdanov, and Y. Togawa, New J. Phys. 18, 095004 (2016).

[46] M. Birch, D. Cortés-Ortuño, L. Turnbull, M. Wilson, F. Groß, N. Träger, A. Laurenson, N. Bukin, S. Moody, M. Weigand et al., Nat. Commun. 11, 1726 (2020).

[47] A. O. Leonov, Y. Togawa, T. L. Monchesky, A. N. Bogdanov, J.-I. Kishine, Y. Kousaka, M. Miyagawa, T. Koyama, J. Akimitsu, T. Koyama, K. Harada, S. Mori, D. McGrouther, R. Lamb, M. Krajnak, S. McVitie, R. L. Stamps, and K. Inoue, Phys. Rev. Lett. 117, 087202 (2016).

[48] A. Vansteenkiste, J. Leliaert, M. Dvornik, M. Helsen, F. GarciaSanchez, and B. Van Waeyenberge, AIP Adv. 4, 107133 (2014).

[49] R. Takagi, D. Morikawa, K. Karube, N. Kanazawa, K. Shibata, G. Tatara, Y. Tokunaga, T. Arima, Y. Taguchi, Y. Tokura, and S. Seki, Phys. Rev. B 95, 220406(R) (2017).

[50] J. Müller, J. Rajeswari, P. Huang, Y. Murooka, H. M. Rønnow, F. Carbone, and A. Rosch, Phys. Rev. Lett. 119, 137201 (2017).

[51] J. C. Loudon, A. Leonov, A. Bogdanov, M. C. Hatnean, and G. Balakrishnan, Phys. Rev. B 97, 134403 (2018).

[52] F. N. Rybakov, A. B. Borisov, S. Blügel, and N. S. Kiselev, New J. Phys. 18, 045002 (2016).

[53] F. N. Rybakov, A. B. Borisov, S. Blügel, and N. S. Kiselev, Phys. Rev. Lett. 115, 117201 (2015).

[54] F. Zheng, F. N. Rybakov, A. B. Borisov, D. Song, S. Wang, Z.-A. Li, H. Du, N. S. Kiselev, J. Caron, A. Kovács et al., Nat. Nanotechnol.1 (2018).

[55] A. S. Ahmed, J. Rowland, B. D. Esser, S. R. Dunsiger, D. W. McComb, M. Randeria, and R. K. Kawakami, Phys. Rev. Mater. 2, 041401 (2018).

[56] G. P. Müller, F. N. Rybakov, H. Jónsson, S. Blügel, and N. S. Kiselev, Phys. Rev. B 101, 184405 (2020).

[57] A. O. Leonov, A. N. Bogdanov, and K. Inoue, Phys. Rev. B 98, 060411(R) (2018).

[58] S. M. Vlasov, V. M. Uzdin, and A. O. Leonov, J. Phys.: Condens. Matter 32, 185801 (2020).

[59] V. Ukleev, Y. Yamasaki, D. Morikawa, K. Karube, K. Shibata, Y. Tokunaga, Y. Okamura, K. Amemiya, M. Valvidares, H. Nakao et al., Phys. Rev. B 99, 144408 (2019). 